\title{
Canons and Sparrows II*: The Enhanced Bernoulli Exact Method for Determining Statistical Significance and Effect Size in the Meta-Analysis of k 2 × 2 Tables
}

Lawrence Marc Paul ( $\sim$ Impaul@sciencesupportconsulting.com )

Bell Laboratories

\section{Methodology}

Keywords: Meta-analysis, Categorical Analysis, Dichotomous Analysis, Sparse Tables, Mantel-Haenszel, DerSimonian, Exact Solution, Inverse Variance, Convolution, Heterogeneity, Rare Events

Posted Date: January 7th, 2021

DOl: https://doi.org/10.21203/rs.3.rs-139437/v1

License: (c) (1) This work is licensed under a Creative Commons Attribution 4.0 International License. Read Full License

Version of Record: A version of this preprint was published at Emerging Themes in Epidemiology on August 3rd, 2021. See the published version at https://doi.org/10.1186/s12982-021-00101-8. 
Non-parametric exact tests for meta-analysis of categorical data

1

2

3

4

5

6

7

8

9

10

11

12

13

14

15

16

17 Bell Laboratories, Retired

18

19 E-mail: lmpaul@sciencesupportconsulting.com

20

21

22
Lawrence M. Paul 
A non-parametric exact test for meta-analysis of categorical data

23 Running head: Non-parametric exact tests for meta-analysis of categorical data

$24 *$ "Little experience is sufficient to show that the traditional machinery of statistical

25 processes is wholly unsuited to the needs of practical research. Not only does it take a

26 cannon to shoot a sparrow, but it misses the sparrow. The elaborate mechanism built on

27 the theory of infinitely large samples is not accurate enough for simple laboratory data."

28 (R. A. Fisher, 1925)

30 ABSTRACT

32 Background: The use of meta-analysis to aggregate the results of multiple studies has

33 increased dramatically over the last 40 years. For homogeneous meta-analysis, the

34 Mantel-Haenszel technique has typically been utilized. In such meta-analyses, the effect

35 size across the contributing studies of the meta-analysis differ only by statistical error. If

36 homogeneity cannot be assumed or established, the most popular technique developed to

37 date is the inverse-variance DerSimonian \& Laird (DL) technique [1]. However, both of

38 these techniques are based on large sample, asymptotic assumptions. At best, they are

39 approximations especially when the number of cases observed in any cell of the

40 corresponding contingency tables is small.

41 Results: This research develops an exact, non-parametric test for evaluating statistical

42 significance and a related method for estimating effect size in the meta-analysis of $k 2 \times 2$

43 tables for any level of heterogeneity as an alternative to the asymptotic techniques.

44 Monte Carlo simulations show that even for large values of heterogeneity, the Enhanced 
A non-parametric exact test for meta-analysis of categorical data

45 Bernoulli Technique (EBT) is far superior at maintaining the pre-specified level of Type I

46 Error than the DL technique. A fully tested implementation in the R statistical language

47 is freely available from the author. In addition, a second related exact test for estimating

48 the Effect Size was developed and is also freely available.

49 Conclusions: This research has developed two exact tests for the meta-analysis of

50 dichotomous, categorical data. The EBT technique was strongly superior to the DL

51 technique in maintaining a pre-specified level of Type I Error even at extremely high

52 levels of heterogeneity. As shown, the DL technique demonstrated many large violations

53 of this level. Given the various biases towards finding statistical significance prevalent in

54 epidemiology today, a strong focus on maintaining a pre-specified level of Type I Error

55 would seem critical.

56 Keywords: Meta-analysis; Categorical Analysis; Dichotomous Analysis; Sparse Tables;

57 Mantel-Haenszel; DerSimonian; Exact Solution; Inverse Variance, Convolution,

58 Heterogeneity, Rare Events

59

\section{BACKGROUND}

61

62 The use of meta-analysis in epidemiological research has been increasing at a very rapid

63 rate. A review of the National Library of Medicine's online database ("Pub Med") shows

64 that in 1977 there was only a single research article with the term "meta-analysis" in its

65 title. This number had increased to 138 in 1991, 966 in 2005 and to 17,205 in 2019 (see

66 Figure 1). 


\section{Insert About Here}

69 Fig. 1 Number of articles containing "meta-analysis" in the title by year of 70 publication.

\section{1}

72 Part of this growth may be due to the widespread availability of powerful personal

73 computer software making meta-analysis techniques more feasible to implement. More

74 importantly, the need to draw meaningful conclusions from an aggregation of small

75 studies may help explain this exponential growth.

76 The use of meta-analytic techniques is controversial when the contributing studies are not

77 randomized control trials (RCT). Many researchers feel that it is highly misleading to

78 attempt to combine a series of disparate studies [2] while others maintain that, with

79 proper safeguards, meta-analysis allows an extremely useful pooling of smaller studies

80 [3], [4]. A discussion of the appropriateness of meta-analysis is beyond the scope of this

81 paper. Rather, the focus here will be on minimizing unnecessary error in testing the

82 overall statistical significance of a meta-analysis and in estimating the Effect Size.

\section{Overview of $2 \times 2 \times$ k Categorical Meta-Analysis}

84 The " 2 × 2 x k" categorical meta-analysis paradigm is probably the most frequently

85 encountered situation in meta-analysis. It consists of a series of $k$ contributing studies

86 each described by a 2 x 2 contingency table. Every cell of each 2 x 2 table contains the

87 number of occurrences of an event (e.g., disease cases) for the particular combination of

88 row and column variables. For the sake of illustration, we can associate the two columns 
89 of each table with Disease Manifestation vs. No Disease Manifestation and the two rows

90 with Exposure vs. No Exposure. Table 1 represents the results of one of these $k$ studies

91

Table 1 Typical contributing study (one of $k$ ) in a dichotomous meta-analysis

\begin{tabular}{|c|c|c|c|}
\hline & \multicolumn{2}{|c|}{ Disease Status } & \\
\hline Exposure & $\begin{array}{c}\text { Disease } \\
\text { Manifestation }\end{array}$ & $\begin{array}{c}\text { No Disease } \\
\text { Manifestation }\end{array}$ & Total \\
\hline Exposure & 4 & 96 & 100 \\
\hline No Exposure & 2 & 98 & 100 \\
\hline Total & 6 & 194 & 200 \\
\hline
\end{tabular}

93 In most meta-analyses, there are typically two distinct components: 1) A statistical test

94 of the overall difference between the Exposure and No Exposure groups across the $k$

95 contributing studies and 2) A method to pool the observed differences between groups

96 across the $k$ studies in order to estimate the true difference (the Effect Size).

97 Surprisingly, in recent years, many epidemiologists employing meta-analytic techniques

98 have greatly deemphasized the first component. Borenstein et al. [3] conclude:

99 “. . . However, meta-analysis also allows us to move beyond the question of

100 statistical significance, and address questions that are more interesting and also

101 more relevant." (pp. 11-12).

102 Similarly, Higgins et al. [4] rather dismissively state:

103 "... If review authors decide to present a $\mathrm{p}$ value with the results of a meta-

104 analysis, they should report a precise $\mathrm{p}$ value, together with the $95 \%$ confidence

105 interval” (pp. 371-372). 
A non-parametric exact test for meta-analysis of categorical data

106 A method is developed that maintains the Type I error ("false alarm rate") at the desired

107 level, but which has good power to detect true differences across a large range of event

108 probability, number of contributing studies, sample size and level of heterogeneity. An

109 argument can be made that maintaining the Type I error at a pre-specified level is more

110 important than the power (1 - Type II error rate) to detect true differences between

111 conditions. The framers of modern statistical testing called such errors "Errors of the

112 First Kind" and placed a special emphasis on them. Neyman \& Pearson in 1933 stated:

113 "A new basis has been introduced for choosing among criteria available for

114 testing any given statistical hypothesis, $\mathrm{H}_{0}$, with regard to an alternative $\mathrm{H}_{t}$. If $\Theta_{1}$

115 and $\Theta_{2}$ are two such possible criteria and if in using them there is the same

116 chance, $\varepsilon$, of rejecting $\mathrm{H}_{0}$ when it is in fact true, we should choose that one of the

117 two which assures the minimum chance of accepting $\mathrm{H}_{0}$ when the true hypothesis

118 is $\mathrm{H}_{t .} "$ [5][p. 336]

119 Thus, while Neyman \& Pearson supported the effort to choose criteria that yield the

120 greatest power to detect true differences, this effort is secondary to maintaining a pre-

121 specified level of Type I error. A second exact method is developed to estimate the effect

122 size of any statistically significant finding.

123 "Rare" Events and Meta-Analysis

124 The probability of occurrence of a disease is often categorized as "rare" although no

125 specific definition exists. As an example, Higgins et al. state that "There is no single risk

126 at which events are classified as 'rare' " but gives as examples 1 in a 100 or 1 in a 1,000

127 (see [6], p. 520). An obvious related issue is observing zero cases in one or more cells of 
A non-parametric exact test for meta-analysis of categorical data

128 a contingency table. Table 2 shows the expected cell sizes from various realistic 129 combinations of disease probability and contributing study sample size. 


\section{Table 2 Expected number of disease cases as a function of disease probability and individual study sample size (each arm)}

\begin{tabular}{|c|c|c|c|c|}
\hline \multirow[b]{2}{*}{ Disease/Condition } & \multirow[b]{2}{*}{$\begin{array}{l}\text { Approximate } \\
\text { Disease Prob. }\end{array}$} & \multicolumn{3}{|c|}{$\begin{array}{l}\text { Individual Study Sample Size } \\
\text { (each arm) }\end{array}$} \\
\hline & & 100 & 500 & 1000 \\
\hline $\begin{array}{l}\text { Myocardial Infarction Incidence Rate for } \\
\text { Age } \geq 60 \text { years }\end{array}$ & .011 [7] & 1.1 & 5.5 & 11 \\
\hline $\begin{array}{l}\text { Parkinson's Disease Incidence Rate } \\
\text { (60 -69 Age Group) }\end{array}$ & $.00058[8]$ & .06 & .29 & .58 \\
\hline $\begin{array}{l}\text { Alzheimer's Disease Incidence Rate } \\
\text { (60 -74 Age Group) }\end{array}$ & $.002[9]$ & .2 & 1 & 2 \\
\hline $\begin{array}{l}\text { Lung Cancer Incidence Rate } \\
\text { for White Males }\end{array}$ & .00051 [10] & .05 & .26 & .51 \\
\hline
\end{tabular}

\section{0}

131 Table 2 supports the notion that "rare" events are a focus of many epidemiological

132 studies.

133 For homogeneous meta-analysis (i.e. where the effect across studies may be assumed to

134 be the same within statistical variation), the two techniques typically used for categorical

135 data are the Mantel-Haenszel and Peto techniques. Both of these techniques rely on the

136 Mantel-Haenszel Chi Square to test for the overall statistical significance. For

137 heterogeneous meta-analyses, the asymptotic DerSimonian-Laird (DL) inverse variance

138 technique is typically used [1].

139 The problem in applying large sample asymptotic techniques to meta-analyses involving

140 small numbers of cases will be illustrated in the older and much more developed domain

141 of homogeneous meta-analyses. Mantel developed what is probably the most widely

142 used technique for homogeneous meta-analyses [11]. In applying his technique, he 
A non-parametric exact test for meta-analysis of categorical data

143 showed that a minimum of approximately five cases was required in each of the 4 cells of

144 each of the $2 \times 2$ tables for each of the $k$ studies comprising the meta-analysis [12]. This

145 is the same heuristic requirement typically used without any particular justification for

146 the simple chi-square test. Mantel \& Fleiss reviewed the options when a reasonable

147 number of cases was not present in all cells:

148 "The investigators could have obtained data from very many more tables to make

149 things more asymptotic for use of $\mathrm{M}-\mathrm{H}$ [note: this is the Mantel-Haenszel

150 technique], or they could readily have applied a more exact procedure for the data

151 at hand" [p. 134].

152 R. A. Fisher made essentially the same plea in 1925 in the preface to the first edition of

153 his well-known Statistical Methods for Research Workers [13]:

154 "Little experience is sufficient to show that the traditional machinery of statistical

155 processes is wholly unsuited to the needs of practical research. Not only does it

156 take a cannon to shoot a sparrow, but it misses the sparrow. The

157 elaborate mechanism built on the theory of infinitely large samples is not accurate

158 enough for simple laboratory data. Only by systematically tackling small sample

159 problems on their merits does it seem possible to apply accurate tests to practical

160 data." 
A non-parametric exact test for meta-analysis of categorical data

161 Both criticisms suggest the use of exact methods to handle the sparseness of the

162 underlying contingency tables at least for the disease examples contained in Table 2. All

163 but two of the combinations of individual study sample size and disease probability

164 shown in Table 2 would yield fewer than five cases per cell leading to violations of the

165 minimum cell size in the Mantel-Haenszel (MH) Chi Square test, and thus the test would

166 be potentially flawed. In addition, these two cases were for sample size equal to 500 and

1671,000 which may not represent many realistic studies. While this limitation of the $\mathrm{MH}$

168 Chi Square test was known to Mantel and others (e.g. [12]), it seems to generally have

169 been forgotten for meta-analysis of $2 \times 2 \times k$ categorical data. The continued use of an

170 asymptotic test in situations not suited for its use is unacceptable given the computer

171 power that is now available to all researchers.

172 Heterogeneity vs. Homogeneity in Meta-Analyses

173 The term "heterogeneity" refers to the fact that studies done at different times and by

174 different researchers might be expected to yield different results. The expectation is that

175 a variable of interest may be dependent, at least in part, to one or more other variables.

176 The meta-analysis researcher, J. P. T. Higgins stated "As Heterogeneity is to be expected

177 in a meta-analysis: it would be surprising if multiple studies, performed by different

178 teams in different places with different methods, all ended up estimating the same

179 underlying parameter." [ [14], p. 158]. While researchers may agree that heterogeneity is

180 to be expected, there is very little agreement on how to quantify this variability. The

181 obvious candidate is $\tau^{2}$, the estimated variability between studies. However, $\tau^{2}$ is not

182 invariant across study designs and its interpretation may not be intuitive. Alternatives 
183 include $I^{2}$, the ratio of the inter-study variability to the total variability and the $Q$ statistic,

184 which is mathematically related to $I^{2}$ (see, e.g., [15] ).

185 In the technique described in this paper, heterogeneity will be mathematically

186 manipulated through $\tau^{2}$ using the logit distribution as developed by Bhaumik et al. [16].

187 Namely:

188

189

190

191

192 Where:

$193 \quad B$ is the Binomial Distribution

$194 \quad N$ is the Normal Distribution

$195 X_{C i}, X_{E i}$ are the observed number of cases in the control and exposure groups

196 respectively of the ith study

$197 p_{C i}, p_{E i}$ are the event probabilities in the control and exposure groups respectively of

198 the ith study

$199 n_{C i}, n_{E i}$ are the sample sizes in the two groups of the $i t h$ study

$200 \quad \mu$ corresponds to the background event (disease) probability in the exposure and

201 control groups

202 $\boldsymbol{\theta}$ corresponds to the overall ratio of the event probabilities for the exposure group

203 relative to the control group 
A non-parametric exact test for meta-analysis of categorical data

$204 \gamma^{2}$ is a variance corresponding to the uncertainty of the observed disease probability in

205 both the exposure and control groups of the $k$ contributing studies.

$206 \tau^{2}$ is a variance corresponding to the heterogeneity or the "heterogeneity parameter" only

207 in the exposure group.

$208 \boldsymbol{\varepsilon}_{1 i}$ is a Normal distribution deviation in background event (disease) probability for both

209 the exposure and control groups of each of the contributing studies.

$210 \varepsilon_{2 i}$ is a Normal distribution deviation in background disease probability due to

211 heterogeneity in the exposure group of each of the contributing studies

212

\section{The Basic Principles of the Dersimonian-Laird (DL) Method}

215 As stated above, this research specifically contrasts an exact method for conducting meta-

216 analyses in $k 2 \times 2$ tables with heterogeneity with the most popular approach which was

217 developed by DerSimonian and Laird (DL) [1].

218 For each contributing study, the DL technique calculates the logarithm of the sample

219 odds ratio and a corresponding estimate of the variance of this measure based on the

220 asymptotic distribution of these logarithms. Adjustments are made for entries in the

221 individual $2 \times 2$ tables that contain a zero-cell count. Equations 5-8 below capture the

222 core DL approach. In Equation 5, an estimate of the interstudy variability, $\boldsymbol{\tau}^{2}$, is first

223 derived from Cochran's Q statistic and the weights assigned to each of the $k$ contributing

224 studies, $\boldsymbol{\omega}_{\boldsymbol{i}}$. These weights are equal to the inverse of the square of the standard error of

225 the estimate of the odds ratio, $\widehat{\boldsymbol{\theta}}_{\boldsymbol{i}}$, in each of the $k$ contributing studies. 


$$
\hat{\boldsymbol{\tau}}^{2}=\frac{Q-(k-1)}{\sum \omega_{i}-\left(\frac{\sum \omega_{i}^{2}}{\sum \omega_{i}}\right)}
$$

230 As shown in Equation 6, a new set of weights, $\boldsymbol{\omega}_{i}^{\prime}$, are then calculated based on the

231 estimated value of $\hat{\boldsymbol{\tau}}^{2}$ from Equation 5 and the standard errors of the contributing studies.

232

233

$$
\omega_{i}^{\prime}=\frac{1}{S E\left(\widehat{\theta}_{i}\right)^{2}+\tau^{2}}
$$

236 These new weights are then used to calculate estimates of both the overall log odds ratio,

$237 \boldsymbol{\theta}_{\boldsymbol{D} \boldsymbol{L}}$ and its standard error as shown in Equation 7 and 8.

$$
\widehat{\boldsymbol{\theta}}_{D L}=\frac{\sum \omega_{i}^{\prime} \widehat{\theta}_{i}}{\sum \omega_{i}^{\prime}}
$$

$$
S E\left(\widehat{\theta}_{D L}\right)=\frac{1}{\sqrt{\sum \omega_{i}^{\prime}}}
$$

244 A test of statistical significance is then based on a large sample normal distribution. The

245 DL technique requires asymptotic assumptions regarding both the Q statistic used to 
A non-parametric exact test for meta-analysis of categorical data

246 estimate the interstudy variability, $\boldsymbol{\tau}^{2}$, and the normal distribution required to test for

247 statistical significance. A more subtle issue is the possibility of distorting correlations

248 between the individual estimates of the effect size for each contributing study, $\boldsymbol{\theta}_{\boldsymbol{i}}$, and

249 the individual weights used for each of these contributing effect sizes.

\section{RESULTS}

251 A Non-Parametric Exact Test of Overall Statistical Significance for Dichotomous

252 Categorical Meta-Analysis

253 Jakob Bernoulli's notion of what is now called a Bernoulli Trial offers the basis for a

254 non-parametric approach to aggregating multiple epidemiological studies based on

255 dichotomous categorical data. The enhancements to the Bernoulli method developed in

256 this paper offer a practical exact method for assessing the overall statistical significance

257 and effect size of a dichotomous meta-analysis.

258 One of the many important contributions of this outstanding 17th century mathematician

259 was the idea of the fixed probability of an event over a sequence of independent trials

260 which led to what is now called Bernoulli Trials and to the related Binomial Distribution.

261 In brief, Bernoulli viewed a set of statistical events as a series of independent coin flips

262 with each flip having a probability $\mathrm{p}$ of obtaining a head and $\mathrm{q}=1-\mathrm{p}$ of obtaining a tail.

263 This hypothetical coin is often treated as a fair coin where both $\mathrm{p}$ and q equal .5. The

264 simplest Bernoulli Trials approach encompasses a series of $n$ flips and answers questions

265 of the type: what is the probability of observing $\mathrm{x}$ heads in $\mathrm{n}$ such flips? (See for example

266 Rosner [17]). In epidemiology, one could consider each of the $k$ contributing studies of a

267 meta-analysis as a single Bernoulli Trial with $\mathrm{p}=.5$. Then the combination of the $k$ 
A non-parametric exact test for meta-analysis of categorical data

268 studies could be analyzed as a binomial distribution. This is the standard Sign Test (see,

269 for example, [18]).

270 For example, for a meta-analysis of 20 studies, if 15 out of 20 studies had more cases in

271 the exposure group than in the control group, we could ask: What is the probability that

27215 or more of the 20 studies could have shown a larger effect in the exposure group

273 strictly by chance alone? If this cumulative probability is less than a pre-specified level

274 of Type I error (e.g. .05), one would reject the null hypothesis and conclude there

275 probably exists a statistically reliable relationship between exposure and the end point

276 used.

277 The principal reason that this approach has seen little use in practical epidemiology is that

278 it suffers from two critical deficits. First, the dichotomous Bernoulli heads vs. tails

279 approach doesn't deal with the third possibility of a tie. The author of this study believes

280 that no truly useful method to date has been offered to deal with those situations when

281 there are an identical number of events in each of the exposure and the control arms of a

282 study other than to discard the study. Second, a truly exact EBT method requires a

283 complete convolution of the frequency distributions of the contributing studies in order to

284 derive the combined frequency distribution. Even for equal sample size, each of the $k$

285 contributing studies could have a different Bernoulli probability, $p$, requiring a full

286 convolution to determine the null distribution of the total number of times there were

$\mathbf{2 8 7}$ more cases in the exposure group relative to the control group across the $k$ contributing

288 studies. Before dealing with the ties problem, the determination of the combined

289 distribution will be outlined. 
A non-parametric exact test for meta-analysis of categorical data

290 Combining the Individual Studies Contributing to the Meta-Analysis. A critical

291 problem is finding a method for combining the individual study binomial distributions of

292 the $k$ contributing studies each with a possibly different $p$ value into an overall frequency

293 distribution.

294 Prior to the widespread availability of computing power, the convolution of a large

295 number of individual binomial distributions was typically handled by approximate

296 methods given the unwieldy nature of the calculations. Even with the advent of available

297 computer power, convolution is still often impractical. As an example, for a meta-

298 analysis involving 24 studies each with a unique binomial distribution $p$, there are over 2

299 million unique combinations of the studies that need to be considered just to calculate the

300 single discrete probability that exactly 12 of the 24 studies have more cases in the

301 exposure group than in the control group. ${ }^{1}$ However, an exact algorithm was laid out in a

302 readily implementable fashion by Butler and Stephens in a 1993 technical report [19]

303 which can easily be implemented even on a personal computer. The algorithm yields the

304 exact probability distribution of the convolution of individual binomial distributions

305 corresponding to the specific studies contributing to the meta-analysis. The method

306 makes use of a recurrence relationship inherent in the binomial distribution which allows

307 the semi-automatic calculation of its probabilities without resort to the simple but

308 overwhelmingly inefficient enumeration of all of the possible combinations of studies.

309 This easily established relationship can be stated as:

\footnotetext{
1 This number of combinations is simply C $(24,12)=2,704,156$.
} 
A non-parametric exact test for meta-analysis of categorical data

$$
P(X=0)=(1-p)^{n} \quad \text { if } j=0
$$

312

313

$$
P(X=j)=\left\{\frac{(n-j+1)}{j}\right\} *\left\{\frac{p}{(1-p)}\right\} * P(X=j-1) \quad \text { if } j \geq 1
$$

\section{4}

315 Figure 2 compares the estimated number of computer executable steps required in the

316 Butler \& Stephens method compared to a traditional convolution.

\section{Insert About Here}

319 Fig. 2 Estimated computer executable steps per Butler \& Stephens vs. traditional 320 convolution.

322 As can be seen, a traditional convolution is only tractable when the number of

323 contributing studies is less than or equal to approximately 20.

324 The Ties Problem. The next problem in adapting the standard Bernoulli Trials

325 technique to practical meta-analysis is a procedure to deal with the situation where there

326 are an identical number of cases in both the exposure and control arms of a study

327 contributing to the meta-analysis. In studies with small sample sizes and/or low disease

328 probabilities, the highest probability tie is typically the " $0 / 0$ " tie in which no cases are

329 observed in either the exposure or the control arms.

330 A first step in dealing with ties is to more clearly define the criteria for a "success". The

331 present EBT approach defines a success as there being a strictly greater number of cases

332 in the exposure group relative to the control group. Under this definition, the same 
A non-parametric exact test for meta-analysis of categorical data

333 number of cases in both arms of the study or more cases in the control arm of the study is

334 considered a "failure". In essence, this is a trinomial situation. There are successes,

335 failures and ties. We are simply combining the failures where there are more cases in the

336 control group relative to the exposure group and tie situations and calling the

337 combination "failures."

338 Equation 9 below forms the basis of the EBT method. The Greek capital letter " $\sqcap$ has

339 been chosen to specify the probabilities of there being more cases in one arm of the study

340 relative to the other to differentiate these parameters from the underlying disease

341 probabilities:

$342 \quad \Pi_{E_{i}}+\Pi_{C_{i}}+\operatorname{prob}(\text { tie })_{i}=1$

343 where:

$344 \Pi_{E_{i}}=$ probability of there being strictly more cases in the exposure group relative to the

345 control group in Study $i$

$346 \Pi_{C_{i}}=$ probability of there being strictly more cases in the control group relative to the

347 exposure group in Study $i$

$348 \operatorname{prob}(t i e)_{i}=$ probability of finding exactly same number of cases in both groups of Study

$349 i$.

350 Assuming that $\boldsymbol{\Pi}_{\boldsymbol{E}_{\boldsymbol{i}}}$ and $\boldsymbol{\Pi}_{\boldsymbol{C}_{\boldsymbol{i}}}$ would be equal under the null hypothesis of no difference

351 between exposure and control groups and rearranging terms, we have:

352

$$
2 \Pi_{E_{i}}+\operatorname{prob}(\text { tie })_{i}=1
$$

353 Solving for $\boldsymbol{\Pi}_{\boldsymbol{E}_{\boldsymbol{i}}}$ we have: 
A non-parametric exact test for meta-analysis of categorical data

354

$$
\Pi_{E_{i}}=\frac{1-p r o b(t i e)_{i}}{2}
$$

355 Thus, the only requirement for calculating the $\boldsymbol{\Pi}_{\boldsymbol{E}_{i}}$ parameter for each contributing study

356 is to first determine the probability of all tie situations for the study.

357 This is a very straightforward procedure. To determine $\operatorname{prob}(\boldsymbol{t i e})_{i}$ for each of the

358 contributing studies, all of the tie situations need to be enumerated and then summed

359 together.

360 As a simple example, assume that Study $i$ has 100 participants in each of its exposure and

361 control arms and that the underlying event (disease) probability $p$ is 01 .

362 The probability that there are no cases among these 100 participants in the exposure arm

363 would then be:

$364 \operatorname{Prob}(0$ cases $)=.01^{0} *(1-.01)^{100}=.9^{100}=.37$

365 Similarly, the probability of there being no cases in the control arm would also be 37 .

366 Thus, the probability of a " 0,0 " tie would be $.37^{2}=.13$ which is surprisingly large.

367 Table 3 lists the probabilities for the first five tie situations and sums these probabilities

368 to determine $\operatorname{prob}(t i e)_{i} \cdot{ }^{2}$

Table 3 Probability of observing exactly the same number of cases in both the exposure and control groups for background event probability equal to .01 and sample size equal to 100 as a function of the number of observed cases

Number of Cases in Each Group

Probability

2 The probabilities for six or more ties decrease to extremely small values. However, in actuality, the EBT method calculates all possible ties in calculating $\operatorname{prob}(\boldsymbol{t i e})_{i}$ 


\begin{tabular}{|c|c|}
\hline 0 & .13 \\
\hline 1 & .137 \\
\hline 2 & .034 \\
\hline 3 & .004 \\
\hline 4 & .0002 \\
\hline 5 & .00001 \\
\hline Total & .309 \\
\hline
\end{tabular}

370 As shown in Table 3, there is over a 30\% probability of obtaining a tie for 0 cases

371 through 5 cases in both the exposure and control groups. Applying Equation [11] to this

372 hypothetical study, we see that, under the null hypothesis of equal probabilities, $\boldsymbol{\Pi}_{\boldsymbol{E}_{\boldsymbol{i}}}$ and

$373 \Pi_{C_{i}}$ are both equal to .35 . Thus, due to ties, the nominal .50 value for $\boldsymbol{\Pi}_{\boldsymbol{E}_{\boldsymbol{i}}}$ and $\boldsymbol{\Pi}_{\boldsymbol{C}_{\boldsymbol{i}}}$ has

374 been greatly reduced.

375 The EBT technique is indeed a "vote counting" method and such methods have been

376 greatly disparaged by Rothman [20] among others. However, unlike a simple Sign Test,

377 the EBT method is based on a reasonable approach to the ties problem and combines the

378 individual $\boldsymbol{P}_{\boldsymbol{E}_{\boldsymbol{i}}}$ values by doing the equivalent of a formal convolution of the frequency

379 distributions of the individual contributing studies.

381 A Non-Parametric Exact Method for the Estimation of Effect Size for Dichotomous

382 Categorical Meta-Analysis

383 Basic Estimation Technique 
A non-parametric exact test for meta-analysis of categorical data

384 A second exact technique was developed to estimate the effect size for dichotomous

385 categorical meta-analysis. As a starting point, one might simply from the ratio of the

386 average observed event probabilities, $\boldsymbol{p}_{\boldsymbol{E}_{\boldsymbol{i}}}$ and $\boldsymbol{P}_{\boldsymbol{C}_{\boldsymbol{i}}}$, in the exposure and control groups

387 respectively of each study and average these ratios across the $k$ contributing studies.

388 This simple approach, however, is highly biased. As shown in the underlying model that

389 is described in Equations 1 - 4, the number of observed "successes" in the exposure and

390 control arms of the $k$ contributing studies each depend on an identical source of variation

391 captured by $\boldsymbol{\varepsilon}_{\mathbf{1} i}$ in the model. The exposure group, however, contains an additional

392 source of variation, captured by $\boldsymbol{\varepsilon}_{2 i}$ in the model. Figure 3 illustrates the problem of

393 estimating the effect size by simply forming the ratio of $\boldsymbol{p}_{\boldsymbol{E}}$ to $\boldsymbol{p}_{\boldsymbol{C}}$.

Insert About Here

395 Fig. 3 Demonstration of inappropriateness of comparing the $p_{E}$ and $p_{C}$

396 distributions to estimate Effect Size.

397

398 Even for the relative risk of 1.0 depicted in the figure, the exposure distribution will have

399 positive excursions that are not compensated for by equally robust negative excursions at

400 least for small (rare) values of event probability.

401 The differential skew of the $\boldsymbol{p}_{\boldsymbol{E}_{\boldsymbol{i}}}$ distribution relative to the $\boldsymbol{p}_{\boldsymbol{C}_{\boldsymbol{i}}}$ distribution was used to

402 address this issue. The additional skew in the exposed group due to the source of $\boldsymbol{\varepsilon}_{\mathbf{2 i}}$ was

$\mathbf{4 0 3}$ estimated by taking the difference between the total exposure group skew and the

404 expected skew from a pure binomial with the same observed event probability. The 
A non-parametric exact test for meta-analysis of categorical data

405 observed average $\boldsymbol{p}_{\boldsymbol{E}}$ across the $k$ contributing studies was then reduced by a factor

406 proportional to this difference in skew levels.

407 Monte Carlo Simulation of The EBT And DL Techniques for Statistical Significance 408 and Effect Size Estimation

409 A series of Monte Carlo simulations was conducted to evaluate the EBT statistical

410 significance test and the effect size estimation techniques and to compare them to the

411 typically used DerSimonian-Laird Inverse Variance technique. The simulation was

412 written and executed in R: A Programming Environment for Data Analysis and Graphics.

413 [21]. The DerSimonian results were calculated using the "meta" package in R.

414 Five levels of relative risk (ratio of exposure group to control group event probability) of

$4151.0,1.25,1.5,1.75$, and 2.0 were crossed with three levels of disease background event

416 probability (.005, .01, and .05), and three levels of sample size (50,100 and 200). Finally,

417 the number of studies entering into each meta-analysis was chosen to be $5,10,20$, or 40

418 studies.

419 In addition, the heterogeneity between the contributing studies, $\tau^{2}$ [Equation 4], was

420 evaluated at 0 (homogeneity), .4, and .8. This last value of .8 represents a very large

421 variance among the studies and was partially chosen to be able to compare the results

422 with previous work ( [22] and [16] ). As an example, at $\tau^{2}=.8$ a nominal exposure group

423 event probability $\boldsymbol{p}_{\boldsymbol{E}}$ of .05 would vary from of .009 to .24 which is almost a 30:1 ratio.

424 Finally, the common variability in both the exposure and control groups represented by

$425 \gamma^{2}$ in Equation 1 was chosen to be .5 to allow direct comparison with earlier work ( [22]

426 and [16]). 
427 The statistical significance and effect size were evaluated using both the EBT and

428 DerSimonian techniques for each replication. All simulation runs were conducted with

42910,000 replications. A value of .05 was used as the pre-specified level of Type I Error.

430 The "Mid-P" technique advocated by Agresti [23] and others was used to determine the $p$

431 values in a less conservative manner leading to more realistic power levels.

433 Results from the Monte Carlo Simulations: Testing Statistical Significance

434 Figures 4 and 5 show the results using the EBT and DL methods respectively.

435 To simplify presentation, only scenarios in which the expected number of cases was

436 greater than or equal to two were utilized. Table 4 shows the included scenarios.

Table 4 Scenarios included in the analysis of statistical significance

\begin{tabular}{|c|c|c|}
\hline $\begin{array}{c}\text { Background Event } \\
\text { Probability }\end{array}$ & Sample Size & $\begin{array}{c}\text { Expected Number of } \\
\text { Observed Cases }\end{array}$ \\
\hline .01 & 200 & 2 \\
\hline .05 & 50 & 5 \\
\hline .05 & 100 & 10 \\
\hline .05 & 200 & 2.5 \\
\hline
\end{tabular}

440 Fig. 4 Power for number of studies, relative risk, and heterogeneity for EBT. A-D

441 correspond to studies equal to 5, 10, 20 and 40. 
444 Fig. 5 Power for number of studies, relative risk, and heterogeneity for DL. A-D

445 correspond to studies equal to 5, 10, 20 and 40.

447 The basic finding was that the EBT method maintained the prespecified level of Type I

448 error for both the homogeneous and heterogeneous scenarios while the DL method had

449 many violations of this level for heterogeneous scenarios. This limitation of the DL

450 method is highlighted in the inserts in Figure 5 which show the power when the Relative

451 Risk is one (i.e. the false alarm rate). For the homogeneous scenario where $\tau^{2}=0$, both

452 the EBT and the DL methods respect the prespecified Type I error level. However, for $\tau^{2}$

$453=.4$ and for $\tau^{2}=.8$, the DL method exhibits large violations of this level. Not

454 unexpectedly, as the number of contributing studies increases, the power for Relative

455 Risk greater than one increases for both the EBT and DL methods for values of Relative

456 Risk of greater than 1.0. A separate analysis showed that the standard deviation of the

457 power estimates in Figures 4 and 5 was less than or equal to .42\% (i.e. .0042).

458 In actuality, comparing the power between the EBT \& DL techniques for event ratios

459 greater than 1.0 is not possible due to the large number of violations of the pre-specified

460 Type 1 Error violations for the DL technique.

461 Figure 6 is a comparison of Type I Error (false alarm rate) for the current EBT technique

462 and the DL technique as a function of heterogeneity $\left(\tau^{2}\right)$.

Insert About Here

464 Fig. 6 Type I error for EBT and DL methods as a function of heterogeneity.

465 As can be clearly seen, the current EBT technique is relatively resistant to the effects of

466 increasing heterogeneity over a very large range. The DL technique, however exhibits a 
A non-parametric exact test for meta-analysis of categorical data

467 monotonically increasing sensitivity to heterogeneity. A related aspect of any meta-

468 analysis technique's ability to perform well in the face of heterogeneity is its resistance to

469 "contamination" from one or a small number of "rogue studies." Since the EBT method

470 does not directly allow such rogue studies to directly affect the test statistic, it should be

471 much more resistant to these distortions.

472 The large costs of discreteness have been studied by Agresti [24] and others.

473 A first cost of discreteness results when the number of contributing studies is small. The 474 general issue of overcoverage is highlighted in Figure 7.

Insert About Here

476 Fig. 7 Interval overcoverage as a function of the number of contributing studies.

478 The overcoverage is greatest for the smallest number of $k$ contributing studies, and

479 generally decreases as the number of contributing studies increases. As Figure 7

480 demonstrates, even an unrealistic level of 500 contributing studies is still associated with

481 a relatively large level of overcoverage. While such discreteness clearly reduces power,

482 it could be argued that a statistically significant finding based on extremely sparse tables

483 and a handful of studies requires stronger evidence. Unfortunately, the majority of meta-

484 analyses consist of fewer than two or three studies as Kontopantelis et al. have shown in

485 their extensive analysis of all meta-analyses in the Cochrane Library [25].

486 Additional Monte Carlo testing was done for unbalanced designs (unequal sample sizes

487 in the exposure and control arms of the contributing studies) and meta-analyses with

488 unequal sample sizes across contributing studies. Table 5 shows the sample sizes for the

489 two groups for a typical unbalanced design in which the control group sample size is 
A non-parametric exact test for meta-analysis of categorical data

490 twice the exposure group sample size. The sum of the two sample sizes across both arms

491 of the study was chosen to be 200 yielding an average sample size of 100 to allow

492 comparison with the balanced designs of Figures 4 and 5.

493

494

\begin{tabular}{|c|c|c|c|c|c|c|c|c|c|c|}
\hline \multicolumn{1}{|c|}{ Table 5 Sample sizes for simulation of unbalanced designs } \\
\hline & \multicolumn{7}{|c|}{ Sumber of studies = 10 } \\
\hline Group & 1 & 2 & 3 & 4 & 5 & 6 & 7 & 8 & 9 & 10 \\
\hline Exposure & 66 & 66 & 66 & 66 & 66 & 66 & 66 & 66 & 66 & 66 \\
\hline Control & 134 & 134 & 134 & 134 & 134 & 134 & 134 & 134 & 134 & 134 \\
\hline
\end{tabular}

495 Table 6 below shows the results of the simulation for heterogeneity values $\tau^{2}=0$ and $\tau^{2}=$

496.8 , Event (“disease”) Probability of .05, Number of Studies = 10, and Sample Size (avg.)

$497=100$ at the same five levels of Relative Risk used above. The simulation run consisted

498 of 10,000 replications as in Figures 4 and 5.

499 
500

\begin{tabular}{|c|c|c|c|c|c|c|c|c|c|c|}
\hline \multicolumn{11}{|c|}{$\begin{array}{c}\text { Table } 6 \text { Power (\%) for the unbalanced design of table } 5 \\
\tau^{2} \text { (heterogeneity) equal to } 0 \text { and } .8 \text {; event probability }=.05 ; \\
\text { Number of studies equal to 10; Sample size (per study arm) equal to } 100\end{array}$} \\
\hline & \multicolumn{10}{|c|}{ Heterogeneity } \\
\hline & \multicolumn{5}{|c|}{ 0 } & \multicolumn{5}{|c|}{.8} \\
\hline & \multicolumn{10}{|c|}{ Risk Ratio } \\
\hline Technique & 1.0 & 1.25 & 1.5 & 1.75 & 2.0 & 1.0 & 1.25 & 1.5 & 1.75 & 2.0 \\
\hline EBT & 2.1 & 14.4 & 43.3 & 71.0 & 88.4 & 4.2 & 11.0 & 21.6 & 35.0 & 46.9 \\
\hline $\begin{array}{c}\text { DerSimonian } \\
\& \\
\text { Laird }\end{array}$ & 2.2 & 16.5 & 57.2 & 87.5 & 97.7 & 11.5 & 24.0 & 41.6 & 59.2 & 72.8 \\
\hline
\end{tabular}

501

502 As the results in Table 6 indicate, the Type I Error (Relative Risk $=1.0$ ) remained below

503 the specified value of five percent for the EBT technique and was far above this point for

504 the DerSimonian technique when the heterogeneity was equal to .8.

505 Table 7 below shows the sample sizes for the exposure and control groups for each of the

506 contributing studies for a design with unequal sample size across the contributing studies.

507 This particular design was chosen as a relatively extreme case. As can be seen, the

508 average sample size for both the groups was maintained at 100 to allow comparison of

509 the simulation results with the equal sample size scenarios of Figures 4 and 5.

510 
Table 7 Sample sizes for simulation of unequal sample size designs Number of studies equal to 10

\begin{tabular}{|c|c|c|c|c|c|c|c|c|c|c|}
\hline \multicolumn{1}{|c|}{ Table 7 Sample sizes for simulation of unequal sample size designs } \\
& \multicolumn{10}{|c|}{ Sumber of studies equal to 10 } \\
\hline & 1 & 2 & 3 & 4 & 5 & 6 & 7 & 8 & 9 & 10 \\
\hline Group & 175 & 25 & 175 & 25 & 175 & 25 & 175 & 25 & 175 & 25 \\
\hline Exposure & 175 & 25 & 175 & 25 & 175 & 25 & 175 & 25 & 175 & 25 \\
\hline Control & 175 & & & & & & & & & \\
\hline
\end{tabular}

\section{1}

512

513 Table 8 below shows the results of the simulation for a heterogeneity values of $\tau^{2}=0$ and

$514 \tau^{2}=.8$, Event (“disease”) Probability of .05, and Sample Size (individual study arm

515 average $)=100$, at the same five levels of Relative Risk as used above. The simulation

516 run consisted of 10,000 replications as in Figures 4 and 5.

517 
518

519

\begin{tabular}{|c|c|c|c|c|c|c|c|c|c|c|}
\hline Numl & $r$ of $s$ & $\begin{array}{l}\text { Table } \\
\text { heter } \\
\text { ies equ }\end{array}$ & $\begin{array}{l}\text { Powe } \\
\text { neity) } \\
\text { to } 10 \text {; }\end{array}$ & $\begin{array}{l}\text { 6) for } \\
\text { ual to } \\
\text { imple }\end{array}$ & $\begin{array}{l}\text { unbal } \\
\text { ad to. } \\
\text { (avg. }\end{array}$ & $\begin{array}{l}\text { ed des } \\
\text { Event } \\
\text { indiv }\end{array}$ & $\begin{array}{l}\text { of ta } \\
\text { babil } \\
\text { al stt }\end{array}$ & $\begin{array}{l}7 \\
.05 ; \\
\text { arm) }\end{array}$ & al to 1 & \\
\hline & & & & & Hete & eneity & & & & \\
\hline & & & $\mathbf{0}$ & & & & & .8 & & \\
\hline & & & & & & Ratio & & & & \\
\hline Technique & 1.0 & 1.25 & 1.5 & 1.75 & 2.0 & 1.0 & 1.25 & 1.5 & 1.75 & 2.0 \\
\hline EBT & 2.0 & 14.9 & 43.0 & 70.0 & 87.7 & 4.3 & 11.4 & 22.4 & 34.5 & 47.9 \\
\hline $\begin{array}{c}\text { DerSimonian } \\
\& \\
\text { Laird }\end{array}$ & 2.4 & 16.6 & 56.6 & 87.2 & 97.8 & 11.4 & 25.1 & 41.6 & 58.2 & 73.5 \\
\hline
\end{tabular}

520

521 Most importantly, the EBT Technique was superior at protecting the pre-specified level

522 of Type I Error relative to the DL technique at a heterogeneity level of .8.

523 A clear finding of the Monte Carlo simulations common to both meta-analysis techniques

524 studies is the apparent fruitlessness of searching for small effect sizes. Both the EBT and

525 DL techniques are very poor at reliably finding statistically significant results until the

526 relative risk approaches 2.0. While this finding does not directly bear on the issues

527 studied in this report, it does serve as a cautionary tale to those who continue to try to

528 tease out very small effects especially from sparse data.

529 Results from the Monte Carlo Simulations: Effect Size Estimation 
A non-parametric exact test for meta-analysis of categorical data

530 Figures 8 and 9 capture the basic findings for estimating the Effect Size.

531

Insert About Here

533 Fig. 8 Effect Size as function of Relative Risk and heterogeneity. A \& B correspond 534 to the EBT and DL methods.

536 Again, only simulation scenarios in which the expected number of observed cases was 537 greater than or equal to two were utilized. Since the effect of the number of studies

538 contributing to the meta-analysis was small for this effect size estimation, results were

539 averaged across this variable. Figure 8 shows the results for the EBT method and Figure

5409 for the DL method. As shown in the figures, both methods were reasonably successful

541 at estimating the levels of relative risk. However, the EBT method generally

542 underestimated the relative risk for $\tau^{2}=0$ and overestimated it for $\tau^{2}=.8$ while the DL

543 method tended to underestimate the relative risk. Finally, the interquartile range for the

544 DL method was considerably smaller than for the EBT method as shown in Figures 10 545 and 11.

Insert About Here

548 Fig. 9 Semi-Interquartile Range as function of Relative Risk and heterogeneity. A $549 \&$ B correspond to the EBT and DL methods. 
A non-parametric exact test for meta-analysis of categorical data

553 This research has developed an exact test for the meta-analysis of dichotomous,

554 categorical data and a related method to estimate the size of the effect.

555 The Enhanced Binomial Technique (EBT) to Assess Statistical Significance

556 The EBT technique was greatly superior to the DerSimonian technique in maintaining a

557 pre-specified level of Type I Error. As shown, the DerSimonian technique demonstrated

558 many large violations of this level when heterogeneity was present. Given the various

559 biases towards finding statistical significance prevalent in epidemiology today, a strong

560 focus on maintaining a pre-specified level of Type I Error would seem critical (see, e.g.,

561 [26] ). The EBT approach is greatly superior at maintaining this pre-specified value of

562 Type I Error in the face of even extreme heterogeneity.

563

564 The Enhanced Binomial Technique (EBT) to Estimate Effect Size

565 A related but separate method was developed to estimate the effect size. This new

566 technique was comparable to the often-used DL method although both methods

567 demonstrated some accuracy issues. The DL method exhibited a somewhat smaller

568 Semi-IQR variability. The fact that the EBT method was clearly superior in assessing

569 statistical significance while the DL method demonstrated a smaller variability in

570 estimating effect Size supports the possible utility of separating these two procedures as

571 outlined at the beginning of this article. One possibility is to use the EBT and DL

572 methods for statistical significance assessment and effect size estimation respectively.

573

574 While statistical programs providing exact solutions already exist such as Cytel's

575 StatXact, they are beyond the means of most practicing statisticians and epidemiologists. 
A non-parametric exact test for meta-analysis of categorical data

576 For example, Cytel Inc. currently lists a price of over \$900 USD for their current version,

577 StatXact $11[27]$

578 The techniques developed here are written in the almost universal statistical language of

$579 \mathrm{R}$ and are freely available from the author. As such, it is hoped that other researchers

580 would be able to extend and improve these initial versions.

581 As outlined in this report, the use of meta-analysis in epidemiology is increasing very

582 rapidly and appears to be meeting an important need. Fortunately, inexpensive and

583 readily available computer power has also vastly increased in the past forty years. For

584 example, task speed as measured in Million Instructions per Second ("MIPS") has

585 increased from .64 for the IBM370 mainframe computer in 1972 to 238,000 for an Intel

586 Pentium processor personal computer in 2014. [28]. By using the techniques developed

587 here and the computer power available to all researchers today, the determination of

588 statistical significance and the estimation of effect size can be readily accomplished

589 without unnecessary error.

590 Declarations

591 Ethics approval and consent to participate

592 No experimental participants

593

594 Consent for publication

595 No consent required

596

597 Availability of data and material

598 Both software programs are freely available from the author 
599 Competing interests

600 The author has no competing interests

601

$602 \quad$ Funding

603 No funding was obtained for this work

604

605 Authors' contributions

606 Work was fully done by L. Paul

607

608 Acknowledgements

609 No acknowledgements

610

611 REFERENCES

612

1. DerSimonian R, Laird N. Meta-analysis in clinical trials. Controlled Clinical Trials. 1986; 7.3: p. 177-188.

2. Shapiro S. Meta-analysis/Shmeta-analysis. Am J Epidemiol. 1994 Nov 1; 140(9): p. 771-8.

3. Borenstein M, et al.. Introduction to meta-analysis.: John Wiley \& Sons; 2009.

4. Higgins JP, editor. Cochrane handbook for systematic reviews of interventions. Chichester: Wiley-Blackwell; 2008.

5. Neyman J, Pearson ES. On the problem of the most efficient tests of statistical hypotheses. Philosophical Transactions of the Royal Society of London. Series A, Containing Papers of a Mathematical or Physical Character. 1933: p. 289-337. 
6. Higgins J, Deeks JJ, Altman DG. Special topics in statistics. In Higgins J, editor. Cochrane Handbook for Systematic Reviews of Interventions: Cochrane Book Series. Chichester: John Wiley; 2008. p. 481-529.

7. Mons U, Müezzinler A, Gellert C, Schöttker B, Abnet C, Bobak M, et al. Impact of smoking and smoking cessation on cardiovascular events and mortality among older adults: meta-analysis of individual participant data from prospective cohort studies of the CHANCES consortium. bmj. 2015 Apr 20; 350(h1551).

8. Hirsch L, Jette N, Frolkis A, Steeves T, Pringsheim T. The incidence of Parkinson's disease: a systematic review and meta-analysis. Neuroepidemiology. 2016; 46(4): p. 292-300.

9. Alzheimer's Association 2015 Alzheimer's disease facts and figures. Alzheimer's \& dementia. Journal of the Alzheimer's Association. 2015 Mar; 11(3): p. 332.

10. Torre L, Siegel R, Ward E, Jemal A. Global cancer incidence and mortality rates and trends-an update. Cancer Epidemiology and Prevention Biomarkers. 2016 Jan; 25(1): p. 16-27.

11. Mantel N, Haenszel W. Statistical aspects of the analysis of data from retrospective studies of disease. J Natl Cancer Inst. 1959 Apr; 22(4): p. 719-48.

12. Mantel N, Fleiss J. Minimum expected cell size requirements for the Mantel-Haenszel one-degree-of-freedom chi-square test and a related rapid procedure. Am J Epidemiol. 1980; 112(1): p. 129-34.

13. Fisher RA. Statistical methods for research workers. Edinburgh: Oliver and Boyd; 1925.

14. Higgins JPT. Commentary: Heterogeneity in meta-analysis should be expected and appropriately quantified. International Journal of Epidemiology. 2008; 37: p. 1158-1160.

15. Higgins JPT, Thompson SG, Deeks JJ, Altman DG. Measuring inconsistency in meta-analyses. British Medical Journal. 2003; 327(7414): p. 557.

16. Bhaumik DK, Amatya A, Normand SLT, Greenhouse J, Kaizar E, Neelon B, et al. Meta-analysis of rare binary adverse event data. Journal of the American Statistical Association. 2012; 107(498): p. $555-567$.

17. Rosner B. Fundamentals of Biostatistics. 5th ed.: Duxbury Press; 1999. 
18. Siegel S, Castellan NJ. Nonparametric statistics for the behavioral sciences. 2nd ed.; 1988.

19. Butler K, Stephens M. Distribution of a Sum of Binomial Random Variables. Technical Report No. 467 prepared under contract N00014-92-5-1264 (NR-042-267) for The Office of Naval Research. Palo Alto: Stanford University; 1993.

20. Rothman K, Greenland S. Meta-analysis. Some methods to avoid: Qualitative tally (vote counting) and Quality scoring. New York: Lippincott Williams Wilkins; 1998.

21. The R Project for Statistical Computing.. Available from: https://www.rproject.org/.

22. Paul LM. Cannons and sparrows: an exact maximum likelihood nonparametric test for meta-analysis of $\mathrm{k} 2 \times 2$ tables. Emerging themes in epidemiology. 2018 December; 15(1).

23. Agresti A. A survey of exact inference for contingency tables. Statistical science. 1992; 7(1).

24. Agresti A. Dealing with discreteness: makingexact'confidence intervals for proportions, differences of proportions, and odds ratios more exact. Statistical Methods in Medical Research. 2003 Feb; 12(1).

25. Kontopantelis E, Springate D, Reeves D. A re-analysis of the Cochrane Library data: the dangers of unobserved heterogeneity in metaanalyses. PloS one. 2013 Jul 26; 8(7).

26. Ioannidis JPA. Why most published research findings are false. PLoS Med. 2005: p. e124.

27. Cytel Inc. Cytel. [Online].; 2020 [cited 2020 November 17]. Available from:

https://store.cytel.com/products/statxact?hsCtaTracking=be2ed66d9346-4239-ad8b-c19193bfcda0\% 7C40b5c432-854f-4116-a2d2079223b15428.

28. Wikipedia. [Document: "Instructions per Second"].; 2016 [cited 2016 June 3. Available from: https://en.wikipedia.org/wiki/Instructions_per_second. 
Figures

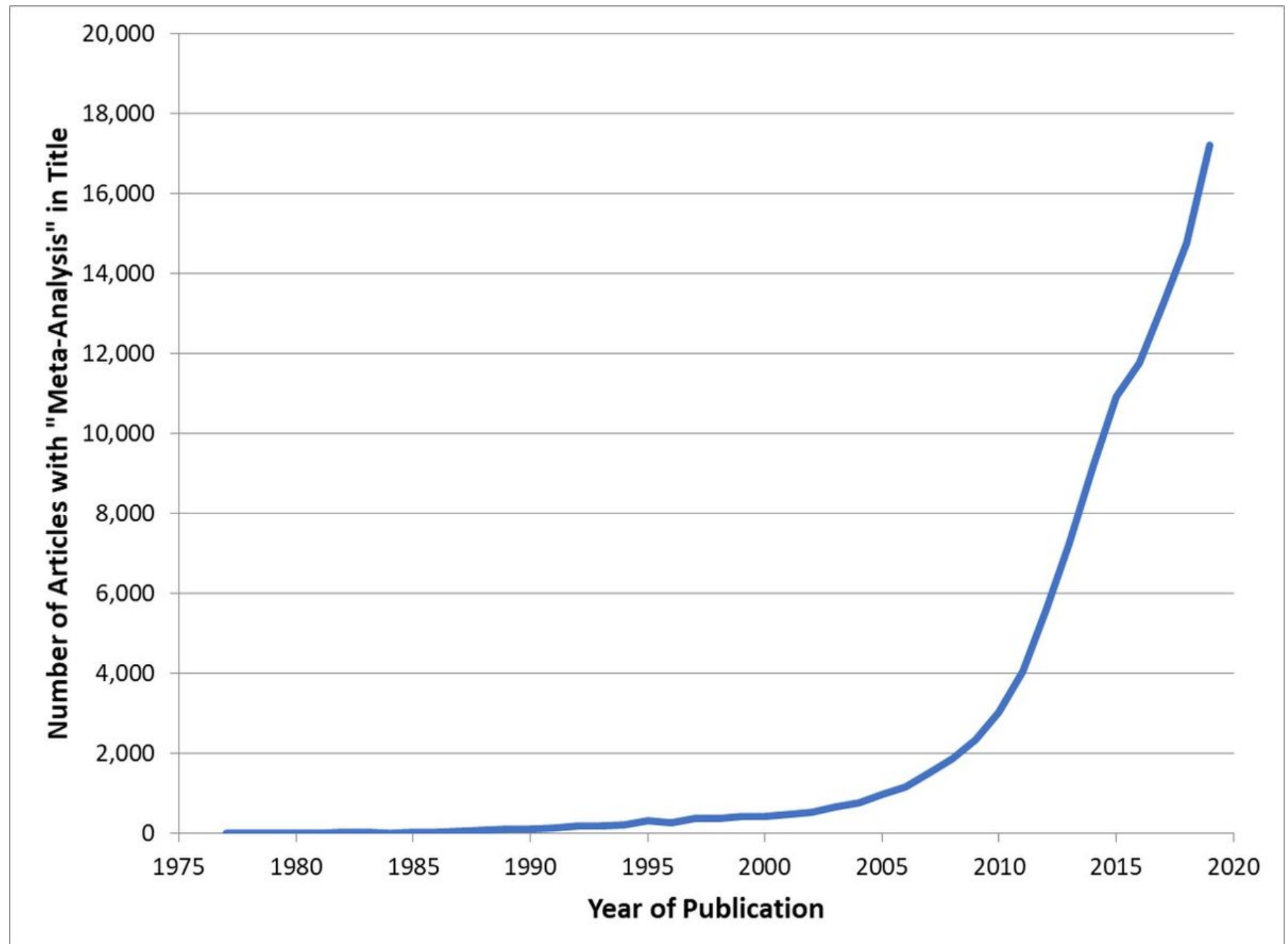

Figure 1

Number of articles containing "meta-analysis" in the title by year of publication. 


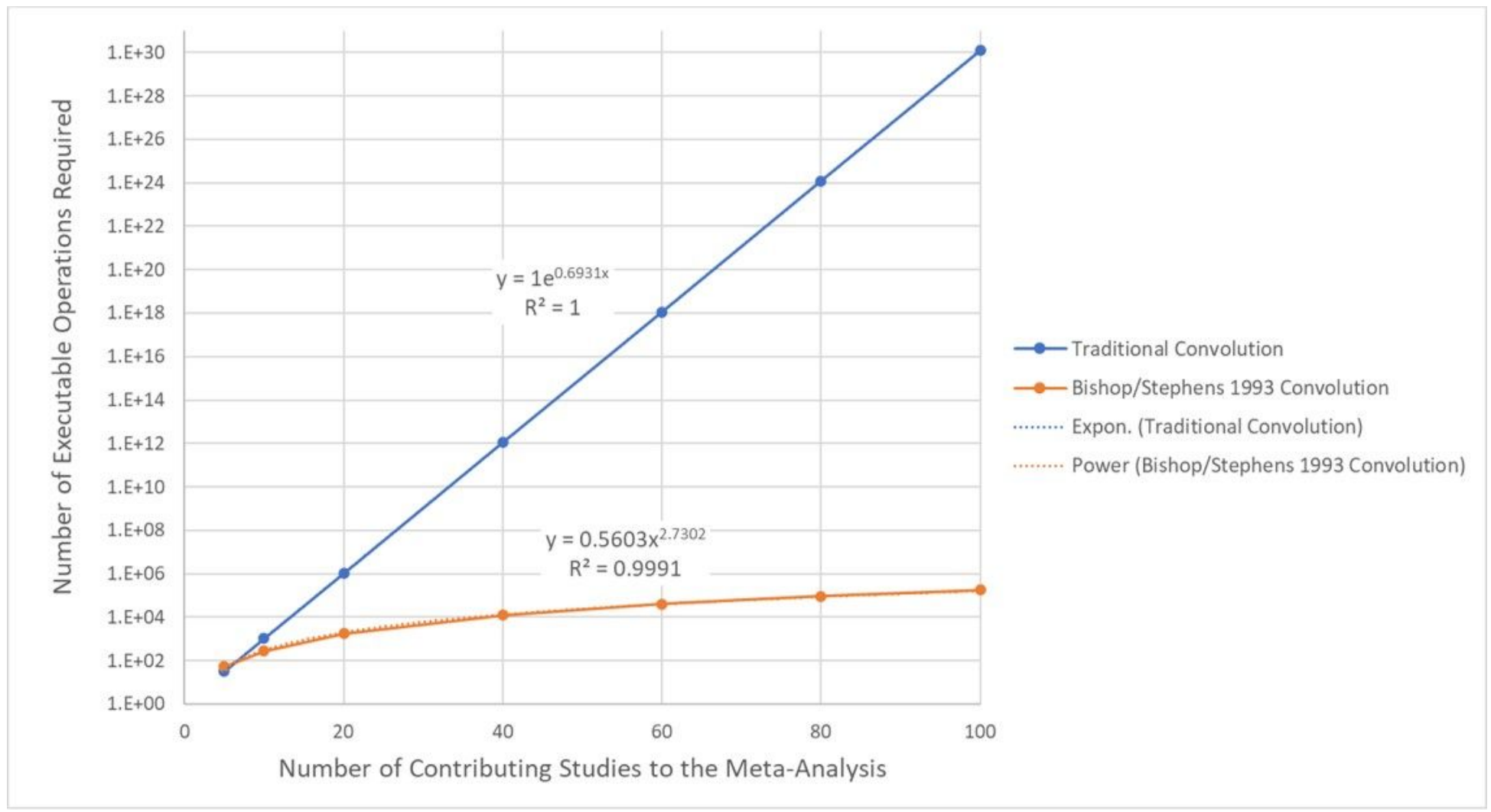

\section{Figure 2}

Estimated computer executable steps per Butler \& Stephens vs. traditional convolution.

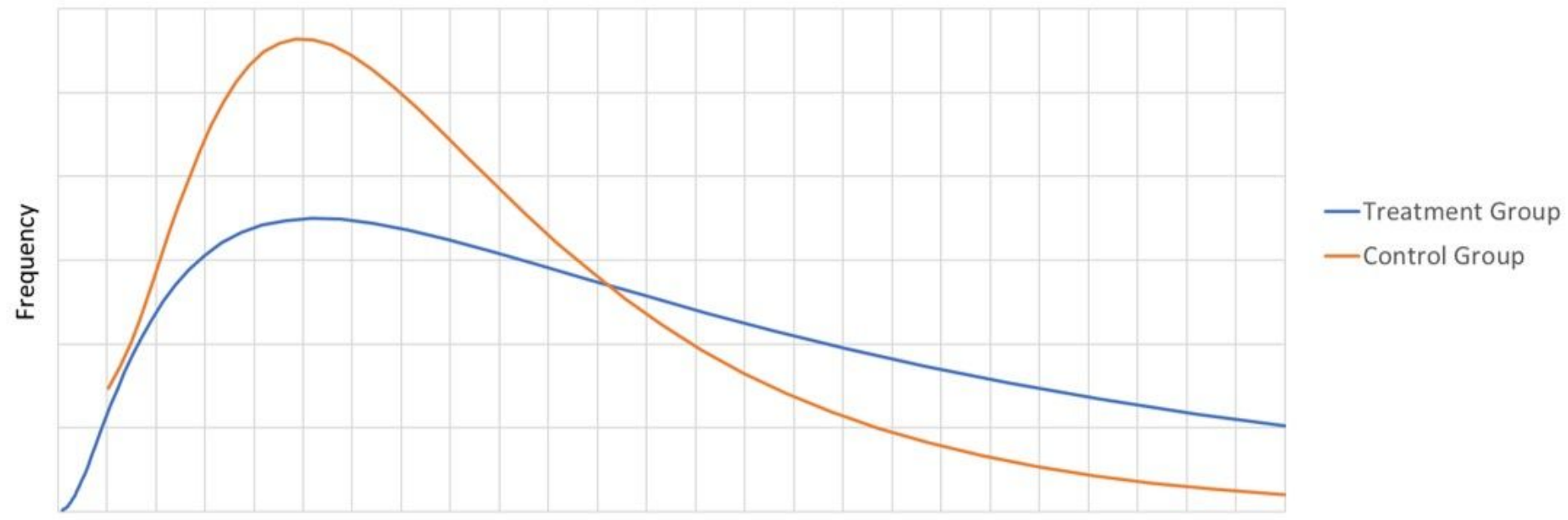

Observed Disease Probability

Figure 3 
Demonstration of inappropriateness of comparing the PE and PC distributions to estimate Effect Size.
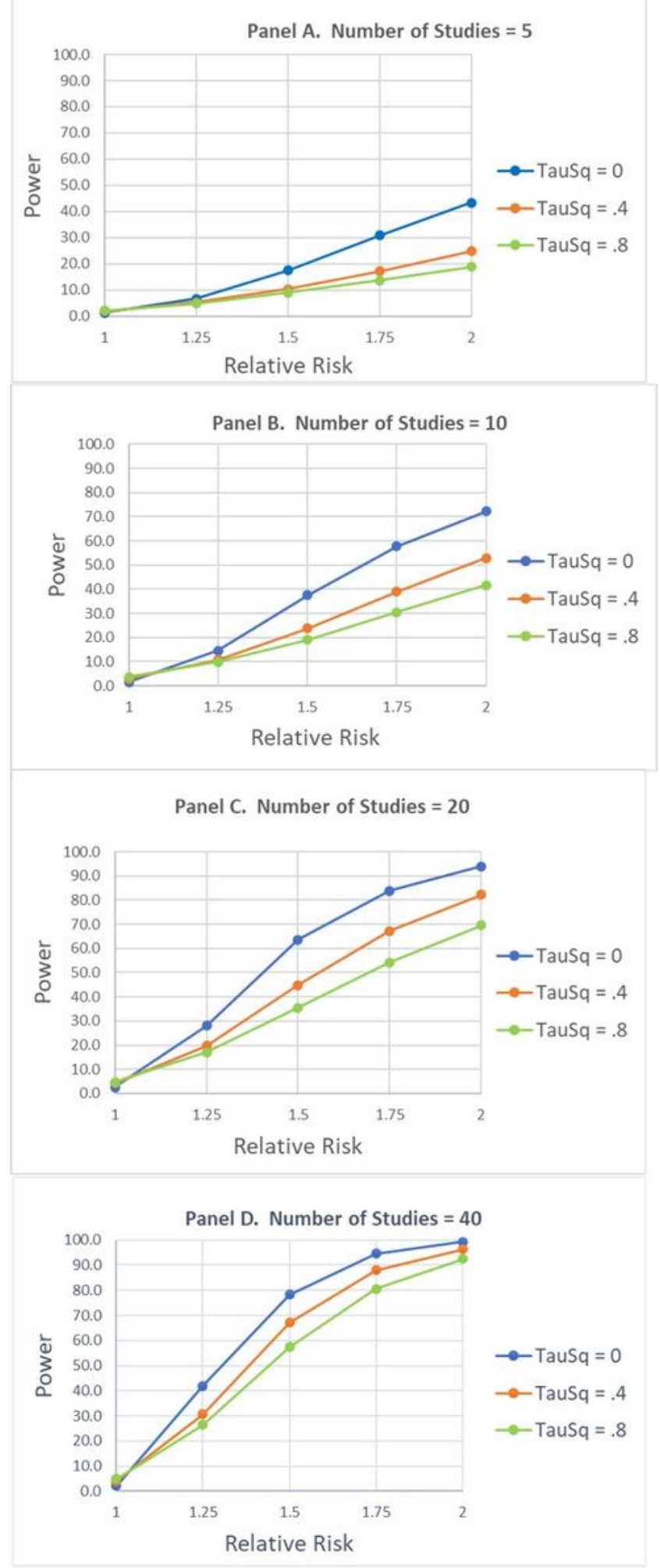

\section{Figure 4}

Power for number of studies, relative risk, and heterogeneity for EBT. A-D correspond to studies equal to 5 , 10,20 and 40. 


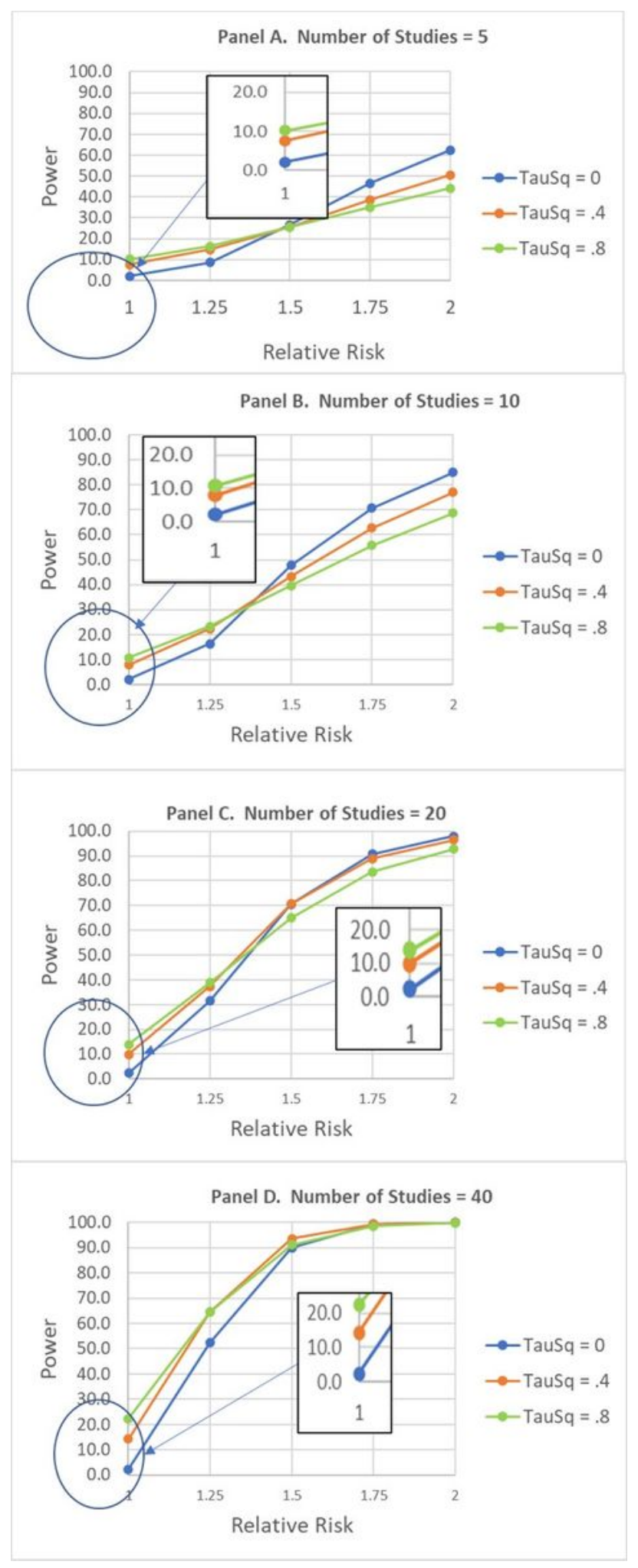

\section{Figure 5}

Power for number of studies, relative risk, and heterogeneity for DL. A-D correspond to studies equal to 5 , 10,20 and 40. 


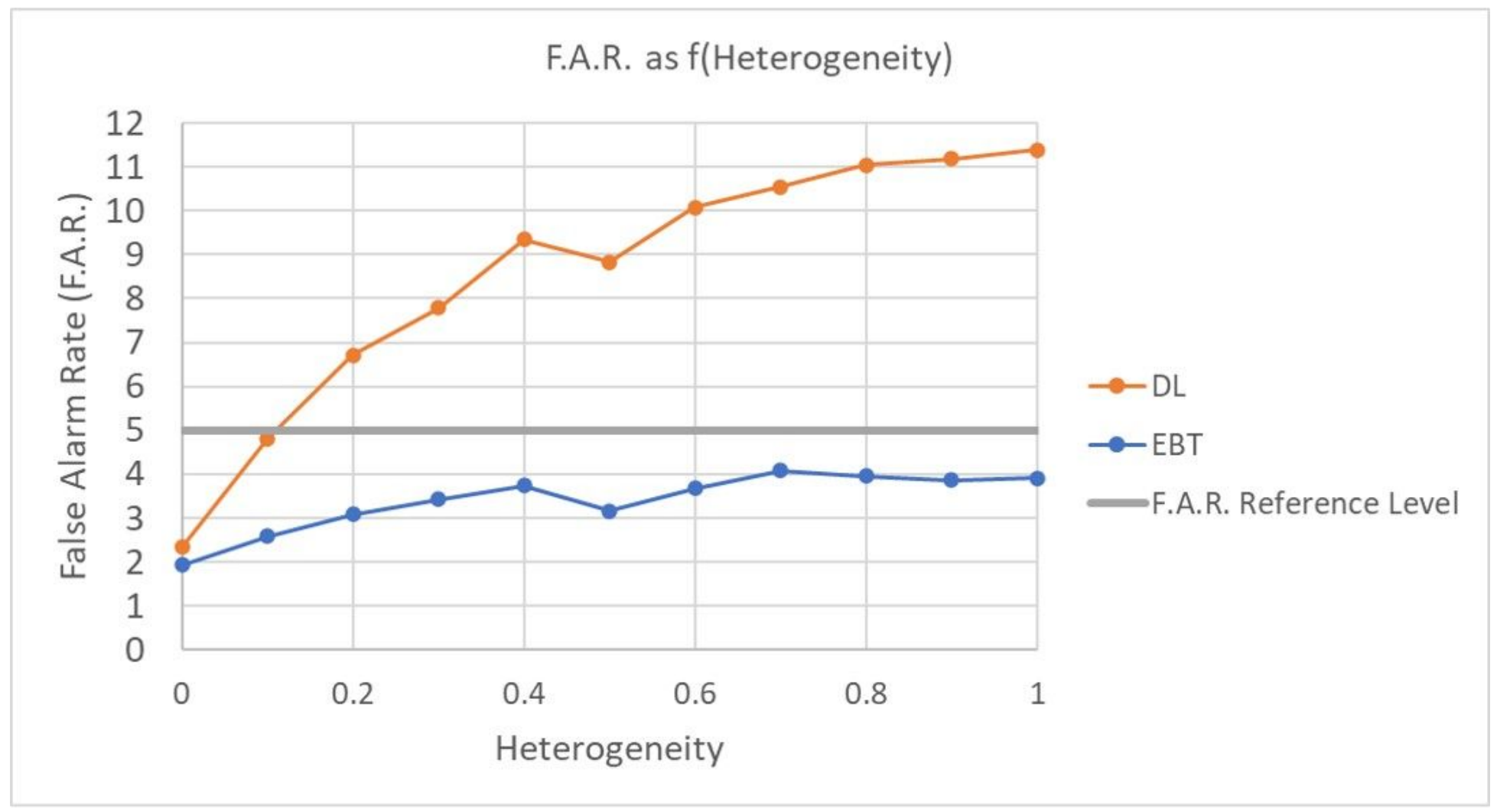

Figure 6

Type I error for EBT and DL methods as a function of heterogeneity. 


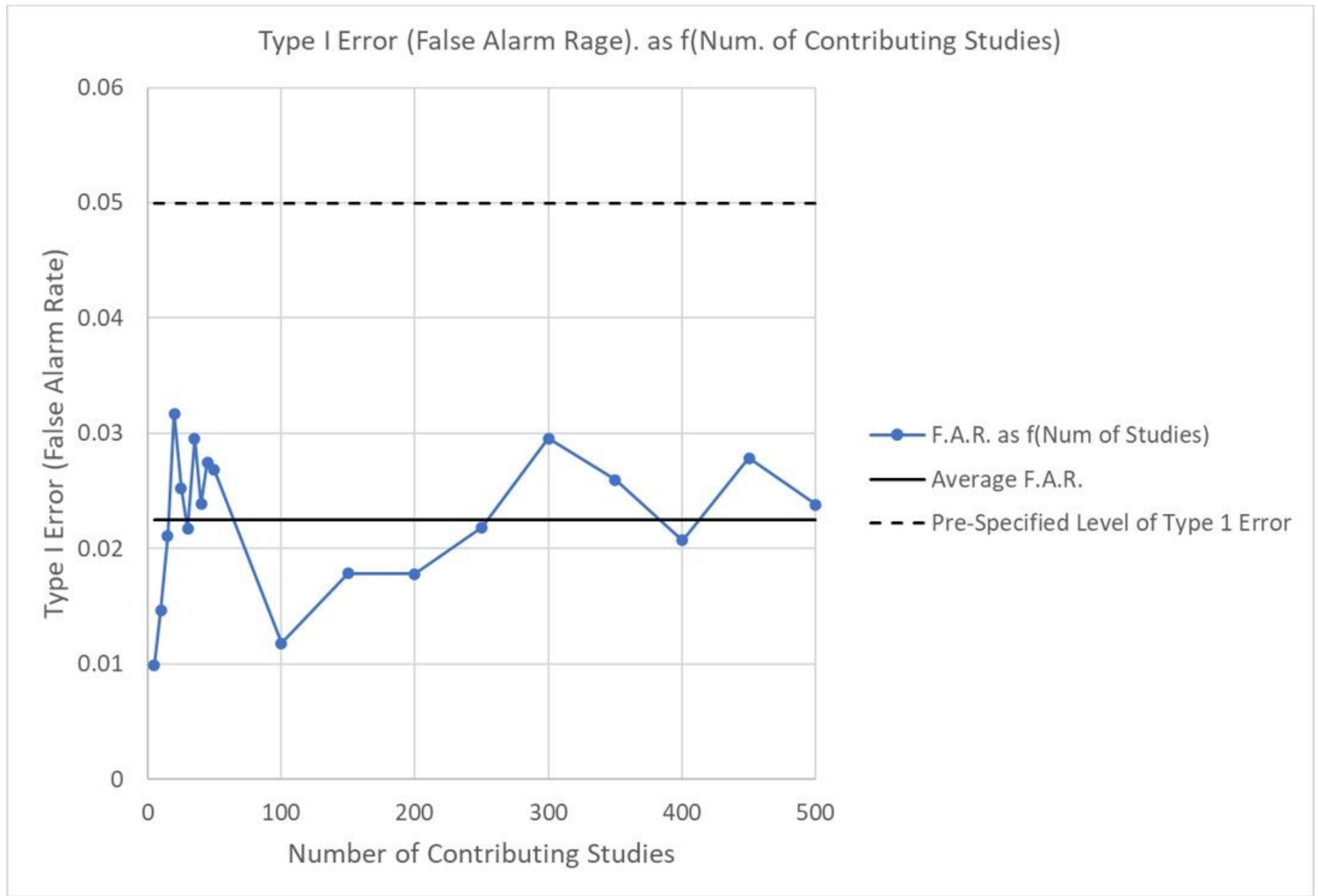

Figure 7

Interval overcoverage as a function of the number of contributing studies. 


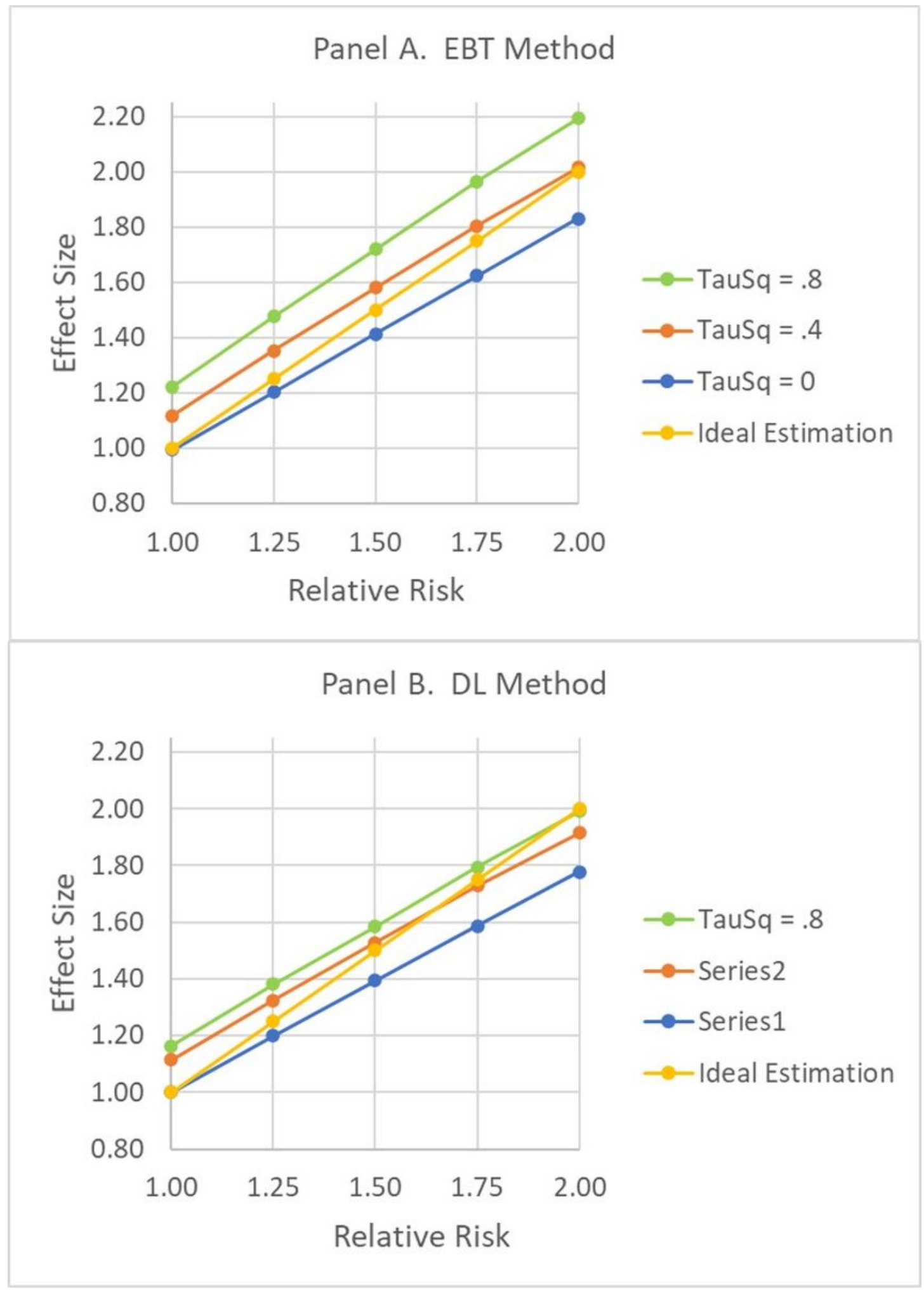

\section{Figure 8}

Effect Size as function of Relative Risk and heterogeneity. A \& B correspond to the EBT and DL methods. 

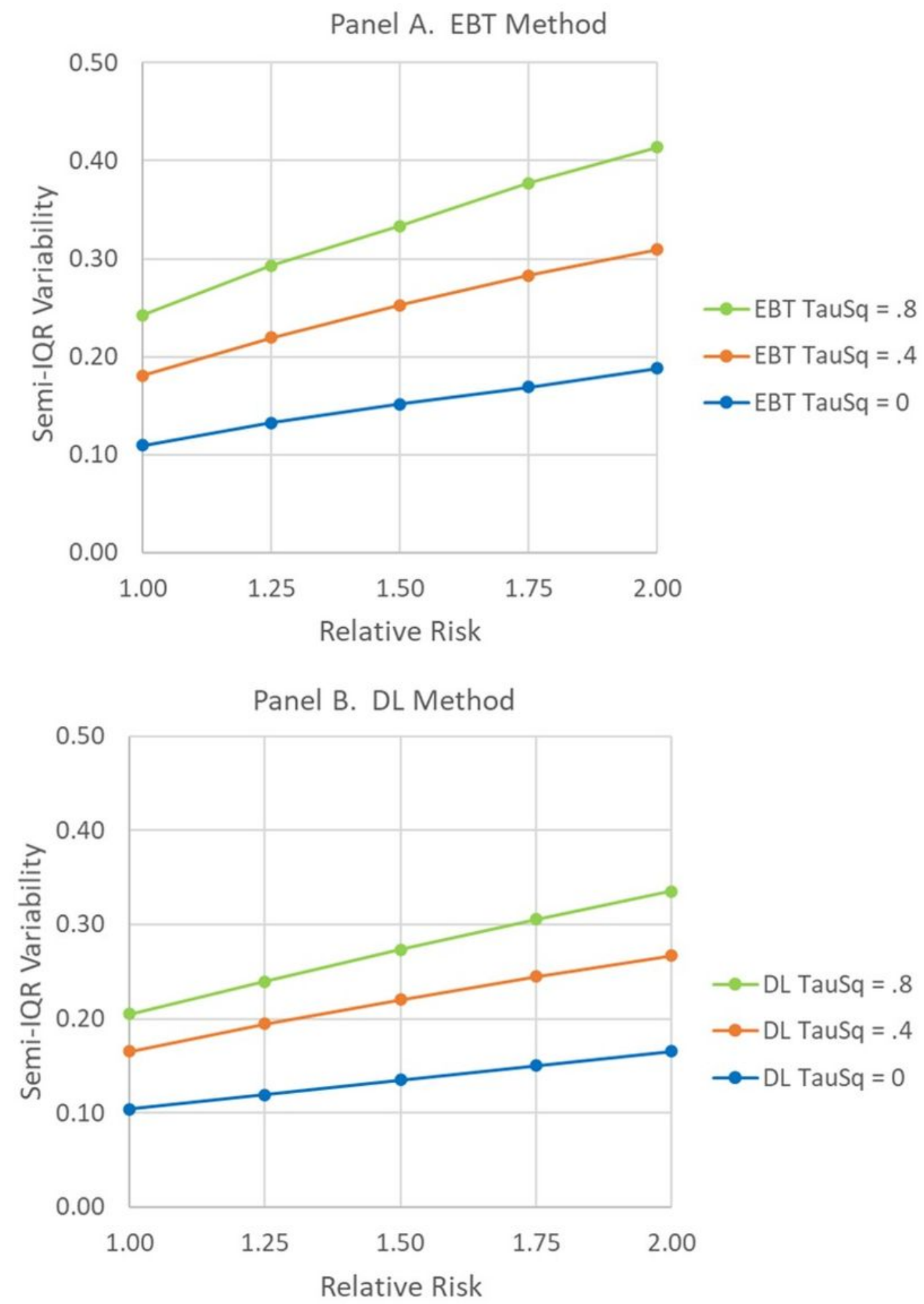

\section{Figure 9}

Semi-Interquartile Range as function of Relative Risk and heterogeneity. A \& B correspond to the EBT and DL methods. 